\title{
Condições climáticas na incidência de cercosporiose (Cercospora coffeicola) e bicho- mineiro (Leucoptera coffeella) em cultivares de cafeeiros em Monte Carmelo, Minas
}

\section{Gerais, Brasil}

Climatic conditions in the cercosporiosis (Cercospora coffeicola) incidence and leaf-miner

(Leucoptera coffeella) in coffee cultivars in Monte Carmelo, Minas Gerais, Brazil

Condiciones climáticas en la incidencia de cercosporiosis (Cercospora coffeicola) y minadores

(Leucoptera coffeella) en cultivares de café en Monte Carmelo, Minas Gerais, Brasil

Recebido: 25/02/2021 | Revisado: 03/03/2021 | Aceito: 09/03/2021 | Publicado: 16/03/2021

Taynara Faria Nascentes

ORCID: https://orcid.org/0000-0003-4025-5027 Universidade Federal de Uberlândia, Brasil

E-mail: taynaranascentes17@gmail.com

Letícia Gonçalves do Nascimento

ORCID: https://orcid.org/0000-0001-9537-5689 Universidade Federal de Uberlândia, Brasil

E-mail: leticia.goncalves5220@gmail.com

Marco Iony dos Santos Fernandes

ORCID: https://orcid.org/0000-0002-2652-6962

Universidade Federal de Uberlândia, Brasil

E-mail: marcoionys@gmail.com

Winícius Baquião Dutra

ORCID: https://orcid.org/ 0000-0001-7560-9986

Universidade Federal de Uberlândia, Brasil

E-mail: winicius.dutra@hotmail.com

Fabio Janoni Carvalho

ORCID: https://orcid.org/0000-0002-0327-1821 Instituto Federal de Educação, Ciência e Tecnologia do Triângulo Mineiro, Brasil

E-mail: fjcarvalho1@gmail.com

Vanessa Andaló

ORCID: https://orcid.org/ 0000-0002-6310-1680

Universidade Federal de Uberlândia, Brasil

E-mail: vanessaandalo@ufu.br

Deyvid da Silva Gallet

ORCID: https://orcid.org/ 0000-0003-4290-2523

Universidade Federal de Uberlândia, Brasi

E-mail: deyvidgallet@hotmail.com

Werik Pereira Dias

ORCID: https://orcid.org/ 0000-0002-7757-8463

Universidade Federal de Uberlândia, Brasil

E-mail: werik.pd@hotmail.com

Gleice Aparecida de Assis

ORCID: https://orcid.org/ 0000-0003-0239-1474

Universidade Federal de Uberlândia, Brasil E-mail: gleice@ufu.br

\section{Resumo}

O material genético adaptado às condições do Cerrado Mineiro e o monitoramento pode ser um fator determinante para o manejo eficiente de pragas e doenças do cafeeiro. Neste contexto, objetivou-se avaliar as condições climáticas na incidência de cercosporiose e bicho-mineiro em cultivares de cafeeiros na região de Monte Carmelo, Minas Gerais. $\mathrm{O}$ experimento foi conduzido na Universidade Federal de Uberlândia, Campus Monte Carmelo. O delineamento experimental utilizado foi em blocos casualizados, com quatro blocos, e os tratamentos foram constituídos por oito cultivares de Coffea arabica L.: Acaiá Cerrado MG-1474; Mundo Novo IAC 379-19; Bourbon Amarelo IAC J10; Catuaí Vermelho IAC 99; Paraíso MG H 419-1; Topázio MG-1190; Acauã Novo e IAC 125 RN. Cada parcela experimental foi constituída por 10 plantas, sendo avaliadas as cinco plantas centrais. Foi avaliada mensalmente a presença de lesões de cercosporiose no ramo localizado no terço superior pelo método não destrutivo. Foram contabilizadas quinzenalmente o número de folhas com minas intactas de bicho-mineiro no ramo plagiotrópico 
selecionado no terço médio. A incidência de cercosporiose e bicho-mineiro foi avaliada no período entre dezembro de 2017 a agosto de 2018. As cultivares que apresentaram menor incidência de bicho-mineiro foram Mundo Novo IAC 379-19, Acaiá Cerrado MG-1474, Bourbon Amarelo IAC J10, Paraíso MG H 419-1 e Topázio MG-1190, respectivamente. A maior incidência de bicho-mineiro ocorreu no período de abril até agosto. Os meses que apresentaram maior incidência de cercosporiose foram de dezembro a maio, havendo variabilidade da ocorrência da doença entre as cultivares testadas.

Palavras-chave: Material genético; Coffea arabica L.; Doença; Praga.

\begin{abstract}
The genetic material adapted to the Cerrado Mineiro conditions and the monitoring can be a determining factor for the efficient management of coffee pests and diseases. In this context, the objective was to evaluate the climatic conditions in the cercosporiosis incidence and leaf-miner in coffee cultivars in Monte Carmelo, Minas Gerais. The experiment was conducted at the Federal University of Uberlandia, Campus Monte Carmelo. The experimental design used was in randomized blocks, with four blocks, and the treatments consisted of eight cultivars of Coffea arabica L.: Acaiá Cerrado MG-1474; Mundo Novo IAC 379-19; Bourbon Amarelo IAC J10; Catuaí Vermelho IAC 99; Paraíso MG H 419-1; Topázio MG-1190; Acauã Novo and IAC 125 RN. Each experimental plot consisted of 10 plants, with the five central plants being evaluated. The presence of cercosporiosis lesions in the branch located in the upper third was evaluated monthly by the non-destructive method. Fortnightly the number of leaves with intact mines of the leafminer in the plagiotropic branch selected in the middle third was counted. The incidence of cercosporiosis and leafminer was evaluated from December 2017 to August 2018. The cultivars that presented the lowest incidence of leafminer were Mundo Novo IAC 379-19, Acaiá Cerrado MG-1474, Bourbon Amarelo IAC J10, Paraíso MG H 419-1 and Topázio MG-1190, respectively. The highest incidence of leaf-miner was from April to August. The months with the highest incidence of cercosporiosis were from December to May, with variability in the occurrence of the disease among the cultivars tested.
\end{abstract}

Keywords: Genetic material; Coffea arabica L.; Disease; Pest.

\title{
Resumen
}

El material genético adaptado a las condiciones del Cerrado Mineiro y la supervisión puede ser un factor determinante para el manejo eficiente de las plagas y enfermedades del café. En este contexto, el objetivo fue evaluar las condiciones climáticas en la incidencia de cercosporiosis y minadores en cultivares de café de la región de Monte Carmelo, Minas Gerais. El experimento se llevó a cabo en la Universidad Federal de Uberlândia, Campus Monte Carmelo. El diseño experimental utilizado fue en bloques al azar, con cuatro bloques, y los tratamientos consistieron en ocho cultivares de Coffea arabica L.: Acaiá Cerrado MG-1474; Mundo Novo IAC 379-19; Bourbon Amarelo IAC J10; Catuaí Vermelho IAC 99; Paraíso MG H 419-1; Topázio MG-1190; Acauã Novo e IAC 125 RN. Cada parte experimental constaba de diez plantas, siendo evaluadas las cinco plantas centrales. La presencia de lesiones de cercosporiosis en la rama ubicada en el tercio superior se evaluó mensualmente por el método no destructivo. Quincenalmente se contó el número de hojas con minas intactas del insecto minero en la rama plagiotrópica seleccionada en el tercio medio. Se evaluó la incidencia de cercosporiosis y mineradores de Minas Gerais en el período de diciembre de 2017 a agosto de 2018. Los cultivares que presentaron menor incidencia de mineradores de Minas Gerais fueron Mundo Novo IAC 379-19, Acaiá Cerrado MG-1474, Bourbon Amarelo IAC J10, Paraíso MG H 419-1 y Topázio MG-1190, respectivamente. La mayor incidencia de insectos mineradores fue de abril a agosto. Los meses con mayor incidencia de cercosporiosis fueron de diciembre a mayo, con variabilidad en la ocurrencia de la enfermedad entre los cultivares probados.

Palabras clave: Material genético; Coffea arabica L.; Enfermedad; Plaga.

\section{Introdução}

A produção de café é considerada uma das atividades mais importantes para a economia brasileira, visto que o Brasil é o maior produtor e exportador mundial, obtendo na safra 2020 uma produção de 63 milhões de sacas beneficiadas (Conab, 2020). A estimativa para a próxima safra é uma produção de 49,59 milhões de sacas beneficiadas, o que representa um decréscimo de $21 \%$ em relação à 2020 devido à bienalidade negativa. O parque cafeeiro brasileiro é formado por cerca de 2,18 milhões de hectares. Minas Gerais destaca-se como o maior produtor do Brasil, responsável por 55\% de todo o café produzido (Coffea arabica L. e C. canephora Pierre ex A. Froehner), representado em sua grande maioria pela produção de $C$. arabica (Conab, 2021).

O Cerrado Mineiro é composto pelas regiões do Alto Paranaíba, Triângulo Mineiro e Noroeste de Minas. O café produzido nesta localidade tem se consolidado com a adoção de tecnologias que proporcionaram a obtenção de elevadas 
produtividades e qualidade superior da bebida (Ortega \& Jesus, 2011).

A produtividade da lavoura foi por um longo tempo, o principal critério de seleção de cultivares de cafeeiros (Carvalho et al., 2010). Entretanto, a presença de inúmeras doenças e pragas que diminuem a produtividade e longevidade da cultura são as principais variáveis consideradas nos programas de melhoramento genético atuais. $\mathrm{O}$ efeito do manejo inadequado, as condições climáticas adversas e outras características agronômicas resultaram em uma pressão pela escolha adequada da cultivar (Silva et al., 2006).

A incidência constante de pragas e doenças é um fator limitante que infere diretamente na escolha da cultivar (Mesquita et al., 2016). Neste sentido, a obtenção de cultivares portadoras de genes de resistência aos patógenos por meio do melhoramento genético constitui uma alternativa economicamente viável e eficiente, além de evitar a contaminação do ambiente e de trabalhadores rurais pela utilização desmedida de produtos fitossanitários (Oliveira \& Ghini, 2012; Alkimin, 2013).

Os programas de melhoramento genético estão desenvolvendo cultivares que sejam resistentes a pragas e doenças visando proporcionar melhor desenvolvimento da planta e produtividade. Algumas cultivares como: Paraíso MG H 419-1, Acauã Novo e IAC 125 RN são resistentes à ferrugem do cafeeiro (Hemileia vastatrix Berk \& Br.) e ao Meloidogyne exigua, apresentando elevadas produtividades na região do Cerrado Mineiro (Fernandes et al.,2020).

A cercosporiose, causada pelo fungo Cercospora coffeicola (Souza et al., 2015) é uma doença que ocorre desde mudas em viveiros até plantas adultas, podendo surgir epidemias severas em lavouras onde práticas adequadas de manejo são negligenciadas. Com relação às pragas, destaca-se o bicho-mineiro, principalmente em regiões com temperaturas mais elevadas e em épocas de estiagem prolongada, podendo ocorrer até 50\% de redução na produção em condições de altos níveis populacionais do inseto (Scalon et al., 2013).

A identificação dos níveis de intensidade do ataque do bicho-mineiro ao longo do ano produtivo é decisiva para o estabelecimento de um programa eficaz no controle do inseto- praga em uma lavoura cafeeira. De posse destas informações, Felipe et al. (2005) afirmam que pode ser estabelecido um programa de manejo fitossanitário capaz de reduzir o impacto ambiental dos produtos fitossanitários, reduzindo o custo de produção e, consequentemente, aumentando o retorno econômico da cafeicultura.

A época de ocorrência de pragas e doenças tem diferido entre as principais regiões cafeeiras, ocorrendo ainda variações em uma mesma região. A ocorrência está condicionada a fatores climáticos, tais como a temperatura e a umidade relativa, sistema de condução da lavoura, presença ou ausência de inimigos naturais, presença ou ausência de plantas daninhas, entre outros. Estes fatores, atuando isolados ou conjuntamente, poderão determinar maiores ou menores infestações (Reis \& Souza, 1996).

Desta forma, as condições climáticas podem influenciar no comportamento das cultivares em relação às doenças e pragas, uma vez que o monitoramento e o material genético adaptado à região são fatores determinantes para o controle de pragas e doenças do cafeeiro. Neste contexto, objetivou-se avaliar as condições climáticas na incidência de cercosporiose e bicho-mineiro em cultivares de cafeeiros na região de Monte Carmelo, Minas Gerais.

\section{Metodologia}

O experimento foi instalado e conduzido na Universidade Federal de Uberlândia, Campus Monte Carmelo. As coordenadas geográficas da área experimental são $18^{\circ} 43^{\prime} 41^{\prime \prime} \mathrm{S}$ e $47^{\circ} 31^{\prime} 26^{\prime \prime} \mathrm{O}$, situada a $903 \mathrm{~m}$ de altitude. As avaliações iniciaram-se no dia 21 de dezembro de 2017 e foram finalizadas no dia 28 de agosto de 2018, totalizando 251 dias.

O solo da área experimental é classificado como Latossolo Vermelho. O plantio foi realizado em janeiro de 2015, com 
espaçamento entre plantas de $0,6 \mathrm{~m}$, totalizando uma área com 320 plantas. Os sulcos de plantio foram abertos a uma distância de $3,5 \mathrm{~m}$ e receberam adubação orgânica de origem bovina na dose de $7,0 \mathrm{~L}_{\text {cova }}{ }^{-1}$ e adubação mineral constituída de superfosfato simples na dose de $195 \mathrm{~g} \mathrm{cova}^{-1}$.

A adubação foi realizada conforme recomendação de Ribeiro, Guimarães \& Alvarez (1999), mediante resultados da análise de solo realizada previamente à instalação do experimento. Trinta dias após o transplantio das mudas, foram aplicados em cobertura $5 \mathrm{~g}$ de $\mathrm{N}$ por planta por aplicação e $5 \mathrm{~g}$ de $\mathrm{K}_{2} \mathrm{O}$ por planta por ano. No primeiro ano após o plantio (dezembro de 2015) foi realizada aplicação de $300 \mathrm{~kg} \mathrm{ha}^{-1}$ de calcário com PRNT igual a $85 \%, 40 \mathrm{~g}$ de N por planta e $10 \mathrm{~g}$ de $\mathrm{K}_{2} \mathrm{O}$ por planta por ano, mediante o parcelamento em quatro vezes, no período de novembro a fevereiro. No segundo ano após o plantio, considerando-se produtividade esperada de 20 a 30 sacas ha $^{-1}$ de $60 \mathrm{~kg}$ de café beneficiado, realizou-se a aplicação de $25 \mathrm{~g}$ planta $^{-1}$ de $\mathrm{N}$ e 12,5 g planta $^{-1}$ de $_{2} \mathrm{O}$, sendo estas parceladas em quatro vezes e aplicadas em intervalos de 30 dias, com início em dezembro de 2016. A adubação fosfatada foi dispensada em função do alto teor deste nutriente no solo. Em dezembro de 2017, referente à segunda safra da lavoura, considerando-se expectativa de produtividade de 50 a 60 sacas ha ${ }^{-1}$ de $60 \mathrm{~kg}$ de café beneficiado, foram aplicados $40 \mathrm{~g}_{\text {planta }}{ }^{-1}$ de $\mathrm{N}$ e $30 \mathrm{~g}_{\text {planta }}{ }^{-1}$ de $\mathrm{K}_{2} \mathrm{O}$, sendo dispensada a adubação fosfatada. Como fontes de adubo foram utilizadas ureia ( $45 \%$ de $\mathrm{N}$ ) e cloreto de potássio vermelho $\left(60 \%\right.$ de $\left.\mathrm{K}_{2} \mathrm{O}\right)$. Ao longo do ciclo da cultura, foram realizadas aplicações foliares de nutrientes em função de deficiências analisadas. A fonte utilizada para tal, de alta solubilidade em água, apresentava $10 \%$ de N, 3\% de $\mathrm{Mg}, 0,4 \%$ de B e $7 \%$ de $\mathrm{Zn}$.

O manejo fitossanitário foi realizado mediante avaliações periódicas na lavoura para determinação da necessidade de manejo de pragas, doenças e plantas daninhas. Para o controle de plantas daninhas em pré-emergência aplicou-se herbicida a base de oxifluorfem, na dose de $2 \mathrm{~L} \mathrm{ha}^{-1}$ (p.c.), além de capinas na projeção da copa do cafeeiro. Para o controle de bichomineiro e broca-do-café (Hypothenemus hampei, Ferrari 1867) foi utilizado inseticida à base de clorantraniliprole e abamectina na dose de $1 \mathrm{~L} \mathrm{ha}^{-1}$ (p.c.) em dezembro de 2017. Para o controle de ferrugem e bicho-mineiro via solo foi aplicado tiametoxam e ciproconazol na dose de $700 \mathrm{~g} \mathrm{ha}^{-1}$ (p.c.) em novembro de 2017. As pulverizações foliares para controle de ferrugem e cercosporiose foram realizadas com fungicida a base de piraclostrobina, na dose de $0,7 \mathrm{~L} \mathrm{ha}^{-1}$ (p.c.).

Os tratamentos foram constituídos por oito cultivares de cafeeiros: Acaiá Cerrado - MG 1474; Mundo Novo IAC 37919; Bourbon Amarelo IAC J10; Catuaí Vermelho IAC 99; Paraíso MG H 419-1; Topázio MG 1190; Acauã Novo e IAC 125 RN. O delineamento utilizado foi em blocos casualizados, com quatro blocos, onde cada parcela experimental foi constituída por uma linha com 10 plantas, considerando como parcela útil as cinco plantas centrais.

A incidência de cercosporiose foi avaliada a cada 30 dias e a avaliação de bicho-mineiro foi realizada a cada quinze dias nas folhas de cinco plantas de cada parcela experimental. O método não destrutivo foi utilizado para as avaliações, consistindo no monitoramento aleatório de um ramo plagiotrópico localizado no terço superior da planta. Em cada ramo selecionado foi realizada a contagem de folhas aleatórias contendo sintomas da doença. Para o bicho-mineiro, foram contabilizadas o número de folhas com minas intactas em um ramo plagiotrópico selecionado no terço médio da planta.

O método utilizado na presente pesquisa foi o quantitativo (Pereira et al., 2018). Os dados de contagem de cercosporiose e bicho-mineiro foram ajustados a um Modelo Linear Generalizado Misto (MLGM) Zero Inflacionado, com distribuição binomial negativa, sendo que as cultivares foram consideradas como efeito fixo e as datas de avaliação como efeito aleatório. Quando diferenças significativas foram detectadas pela Análise de Deviance, por meio do teste de QuiQuadrado ( $<<0,01)$, as médias das cultivares foram comparadas pelo teste de Tukey $(\mathrm{p}<0,05)$ e as médias para cada cultivar foram ajustadas a um Modelo Aditivo Generalizado (MAG) com distribuição binomial negativa, para ajuste de curvas de regressão. 


\section{Resultados e Discussão}

\subsection{Incidência de cercosporiose}

Pela análise de Deviance (Tabela 1), verificou-se efeito significativo dos fatores analisados para incidência de cercosporiose em folhas de cafeeiros. A interação dos fatores cultivares e épocas de avaliação foi significativa $(p<0,001)$. A doença ocorreu em todo o período de avaliação do experimento.

Tabela 1. Análise de Deviance para incidência de cercosporiose em cultivares de cafeeiros avaliadas a cada 30 dias durante os meses de dezembro/2017 a agosto/2018 em Monte Carmelo, Minas Gerais.

\begin{tabular}{cccc}
\hline FV & GL & Deviance & valor- $p$ \\
\hline Cultivar & 7 & $2,8 \times 10^{7}$ & $<0,001^{* *}$ \\
Época & 8 & $1,6 \times 10^{7}$ & $<0,001^{* *}$ \\
Bloco & 3 & $5,3 \times 10^{7}$ & $<0,01^{*}$ \\
Cultivar x Época & 56 & $3,54 \times 10^{7}$ & $<0,001^{* *}$ \\
\hline
\end{tabular}

* significativo ao nível de $1 \%$ de probabilidade, ** significativo ao nível de $0,1 \%$ de probabilidade. FV: Fonte de Variação; GL: grau de liberdade. Fonte: Autores.

Os meses que apresentaram maior incidência foram dezembro, fevereiro, março, abril e maio (Tabela 2). De acordo com Zambolim, Vale e Zambolim (2005) o período de maior incidência da doença, geralmente, ocorre no verão a meados de outono.

Em dezembro de 2017, as cultivares que apresentaram menor incidência média de lesões de cercosporiose foram Topázio MG-1190, IAC 125 RN, Acaiá Cerrado MG-1474 e Acauã Novo. A cultivar Bourbon Amarelo IAC J10 apresentou a maior incidência média (40 lesões a cada cinco ramos avaliados). Essas cultivares vem apresentando resultados significativos à doença (Carvalho et al., 2010; Rodrigues et al., 2015; Carvalho et al., 2017), entretanto, existem dificuldades na obtenção de materiais com tolerância ou resistência ao agente etiológico dessa doença.

No entanto, este resultado contrapõe aos de Patricio, Braguini e Fazuoli (2010) que ao avaliarem a incidência e severidade de cercosporiose em cultivares de cafeeiros observaram que após a inoculação, a incidência média foi de 88,9\%, 76,7\% e 65,6\% em mudas de Mundo Novo, Bourbon Amarelo e Catuaí Vermelho, respectivamente. 


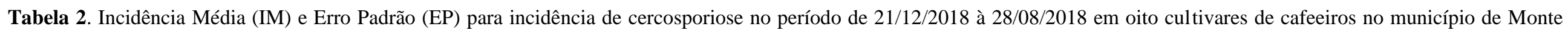
Carmelo, Minas Gerais.

\begin{tabular}{|c|c|c|c|c|c|c|c|c|c|}
\hline Cultivar & $\begin{array}{c}21 / 12 / 2017 \\
\mathrm{IM} \pm \mathrm{EP}\end{array}$ & $\begin{array}{c}21 / 01 / 2018 \\
\mathrm{IM} \pm \mathrm{EP}\end{array}$ & $\begin{array}{c}22 / 02 / 2018 \\
\mathrm{IM} \pm \mathrm{EP}\end{array}$ & $\begin{array}{c}27 / 03 / 2018 \\
\mathrm{IM} \pm \mathrm{EP}\end{array}$ & $\begin{array}{c}27 / 04 / 2018 \\
\mathrm{IM} \pm \mathrm{EP}\end{array}$ & $\begin{array}{c}21 / 05 / 2018 \\
\mathrm{IM} \pm \mathrm{EP}\end{array}$ & $\begin{array}{c}21 / 06 / 2018 \\
\mathrm{IM} \pm \mathrm{EP}\end{array}$ & $\begin{array}{c}28 / 07 / 2018 \\
\mathrm{IM} \pm \mathrm{EP}\end{array}$ & $\begin{array}{c}28 / 08 / 2018 \\
\mathrm{IM} \pm \mathrm{EP}\end{array}$ \\
\hline Mundo Novo & $22,4 a b \pm 9,4$ & $8,3 a b c \pm 3,0$ & $18,6 a b c \pm 8,1$ & $10,0 \mathrm{abc} \pm 4,4$ & $17,0 \mathrm{abc} \pm 7,4$ & $13,0 a b \pm 5,6$ & $4,0 \mathrm{abc} \pm 2,0$ & $2,0 \mathrm{abc} \pm 1,5$ & $1,2 \mathrm{a} \pm 1,5$ \\
\hline $\begin{array}{c}\text { Catuaí } \\
\text { Vermelho }\end{array}$ & $17,3 a b \pm 7,6$ & $7,3 a b c \pm 2,6$ & $23,1 \mathrm{abc} \pm 1,0$ & $12,2 \mathrm{abc} \pm 5,4$ & $10,0 \mathrm{abc} \pm 4,5$ & $14,4 a b \pm 6,4$ & $5,5 \mathrm{abc} \pm 3,0$ & $2,3 a b c \pm 1,7$ & $0,2 \mathrm{a} \pm 4,0$ \\
\hline Acauã Novo & $19,1 \mathrm{a} \pm 1,4$ & $12,0 \mathrm{c} \pm 1,4$ & $18,4 \mathrm{ab} \pm 1,4$ & $11,1 b c \pm 9,1$ & $8,5 a \pm 8,1$ & $21,0 \mathrm{~b} \pm 1,4$ & $3,5 \mathrm{ab} \pm 5,0$ & $6,3 c \pm 4,8$ & $0,5 \mathrm{a} \pm 2,0$ \\
\hline $\begin{array}{l}\text { Bourbon } \\
\text { Amarelo }\end{array}$ & $40,0 \mathrm{~b} \pm 1,2$ & $1,7 \mathrm{a} \pm 1,6$ & $24,4 b c \pm 7,2$ & $17,6 \mathrm{c} \pm 1,3$ & $21,0 \mathrm{c} \pm 1,1$ & $11,5 \mathrm{a} \pm 9,0$ & $3,5 \mathrm{~b} \pm 6,0$ & $1,0 \mathrm{a} \pm 4,4$ & $0,0 \mathrm{a} \pm 1,0$ \\
\hline Paraíso & $21,7 \mathrm{ab} \pm 9,2$ & $9,0 a b c \pm 3,1$ & $17,1 \mathrm{abc} \pm 7,3$ & $16,3 \mathrm{abc} \pm 7,0$ & $30,0 \mathrm{abc} \pm 1,3$ & $13,2 \mathrm{ab} \pm 6,0$ & $0,7 \mathrm{a} \pm 5,7$ & $3,3 \mathrm{abc} \pm 1,5$ & $1,2 \mathrm{a} \pm 1,4$ \\
\hline Acaiá Cerrado & $19,2 \mathrm{a} \pm 9,7$ & $9,0 b c \pm 2,1$ & $22,3 \mathrm{c} \pm 2,0$ & $10,4 b \pm 1,8$ & $13,4 b \pm 9,0$ & $11,0 \mathrm{a} \pm 1,6$ & $6,5 c \pm 3,0$ & $2,5 b \pm 3,4$ & $1,0 \mathrm{a} \pm 7,7$ \\
\hline Topázio & $9,0 \mathrm{a} \pm 5,0$ & $3,7 \mathrm{ab} \pm 1,5$ & $8,7 \mathrm{a} \pm 3,5$ & $2,5 \mathrm{a} \pm 1,5$ & $13,7 \mathrm{abc} \pm 7,0$ & $15,1 \mathrm{ab} \pm 7,7$ & $4,0 \mathrm{abc} \pm 2,2$ & $2,5 a b c \pm 2,0$ & $1,7 \mathrm{a} \pm 2,1$ \\
\hline IAC $125 \mathrm{RN}$ & $10,7 \mathrm{a} \pm 5,3$ & $3,7 a b \pm 1,5$ & $10,4 a b c \pm 5,2$ & $13,0 \mathrm{abc} \pm 7,0$ & $15,5 \mathrm{abc} \pm 8,0$ & $21,4 a b \pm 1,1$ & $1,2 \mathrm{abc} \pm 9,3$ & $4,5 \mathrm{abc} \pm 3,0$ & $0,7 \mathrm{a} \pm 10,0$ \\
\hline
\end{tabular}

Médias seguidas pela mesma letra na coluna não diferem entre si pelo teste Tukey ao nível de 0,01\% de probabilidade. Fonte: Autores. 
Informações sobre as reais condições ambientais que favorecem a doença são genéricas. Para Pozza \& Alves (2008), em ambientes controlados, há um alto favorecimento de ocorrência da cercosporiose em temperaturas mensais médias entre 18 e $25^{\circ} \mathrm{C}$ e precipitação média de $3 \mathrm{~mm} \mathrm{dia}{ }^{-1}$. Portanto, a incidência de cercosporiose está diretamente relacionada com alta umidade relativa e temperatura, excesso de insolação e déficit hídrico (Souza et al., 2013).

As condições nutricionais e hídricas na época das avaliações supriram as necessidades da cultura, não interferindo na avaliação. Deste modo, pode-se afirmar que altas temperaturas combinadas com umidade e precipitação encontradas durante o mês de dezembro favoreceram diretamente a ocorrência da doença (Figura 1).

Figura 1. Dados meteorológicos de Monte Carmelo, Minas Gerais, durante os meses de dezembro de 2017 a agosto de 2018.

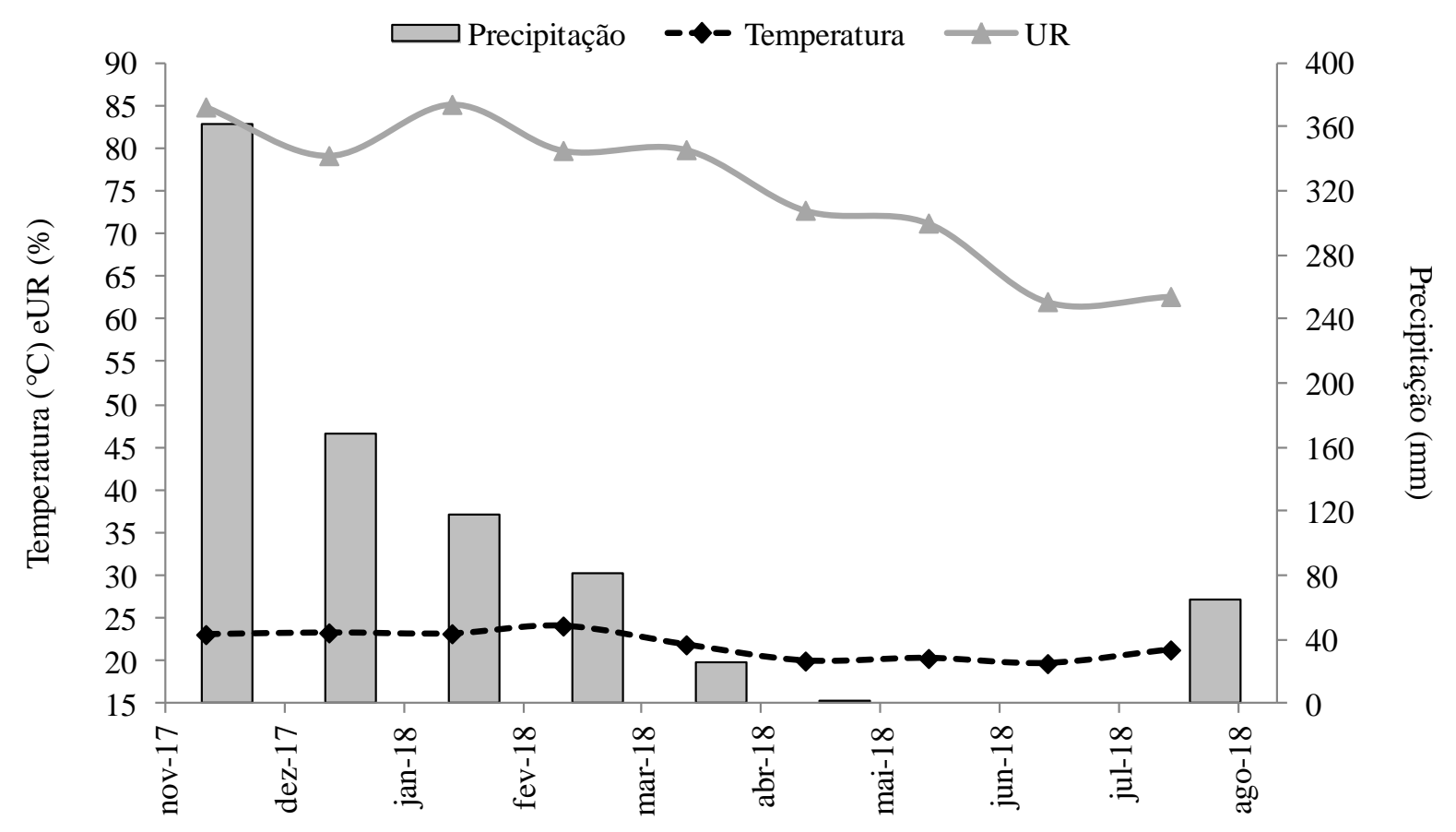

UR - Umidade Relativa, Prec - Precipitação. Fonte: SISMET COOXUPÉ (2018).

O molhamento foliar causado pelas chuvas ou irrigação é um fator indispensável para a ocorrência da doença, pois influenciam positivamente na germinação, infecção e esporulação dos fungos. Silva (2014) observou o progresso da incidência da cercosporiose do cafeeiro, em diversas horas de molhamento foliar e verificou que o maior progresso foi nas condições de temperatura de $25^{\circ} \mathrm{C}$ no tratamento de 72 horas de molhamento foliar. Ademais, cafeeiros cultivados a pleno sol encontram um ambiente físico mais propício ao desenvolvimento da doença do que aqueles cultivados à sombra (Salgado et al, 2007).

O desdobramento do fator épocas dentro de cada cultivar foi realizado por meio de Modelos Aditivos Generalizados (MAG), ajustando-se curvas de regressão para cada material genético analisado (Figura 2). A cultivar Mundo Novo IAC 37919 apresentou, no início das avaliações, alta incidência de cercosporiose. Aos 25 dias de avaliações, houve um decréscimo na ocorrência da mesma, permanecendo constante até os 140 dias. 
Figura 2. Comportamento da incidência de cercosporiose em cultivares de cafeeiros: A) Mundo Novo IAC 379-19; (B) Catuaí Vermelho IAC 99; (C) Acauã Novo; (D) Bourbon Amarelo IAC J10; (E) Paraíso MG H 419-1; (F) Acaiá Cerrado MG-1474; (G) Topázio MG-1190 e (H) IAC 125 RN.
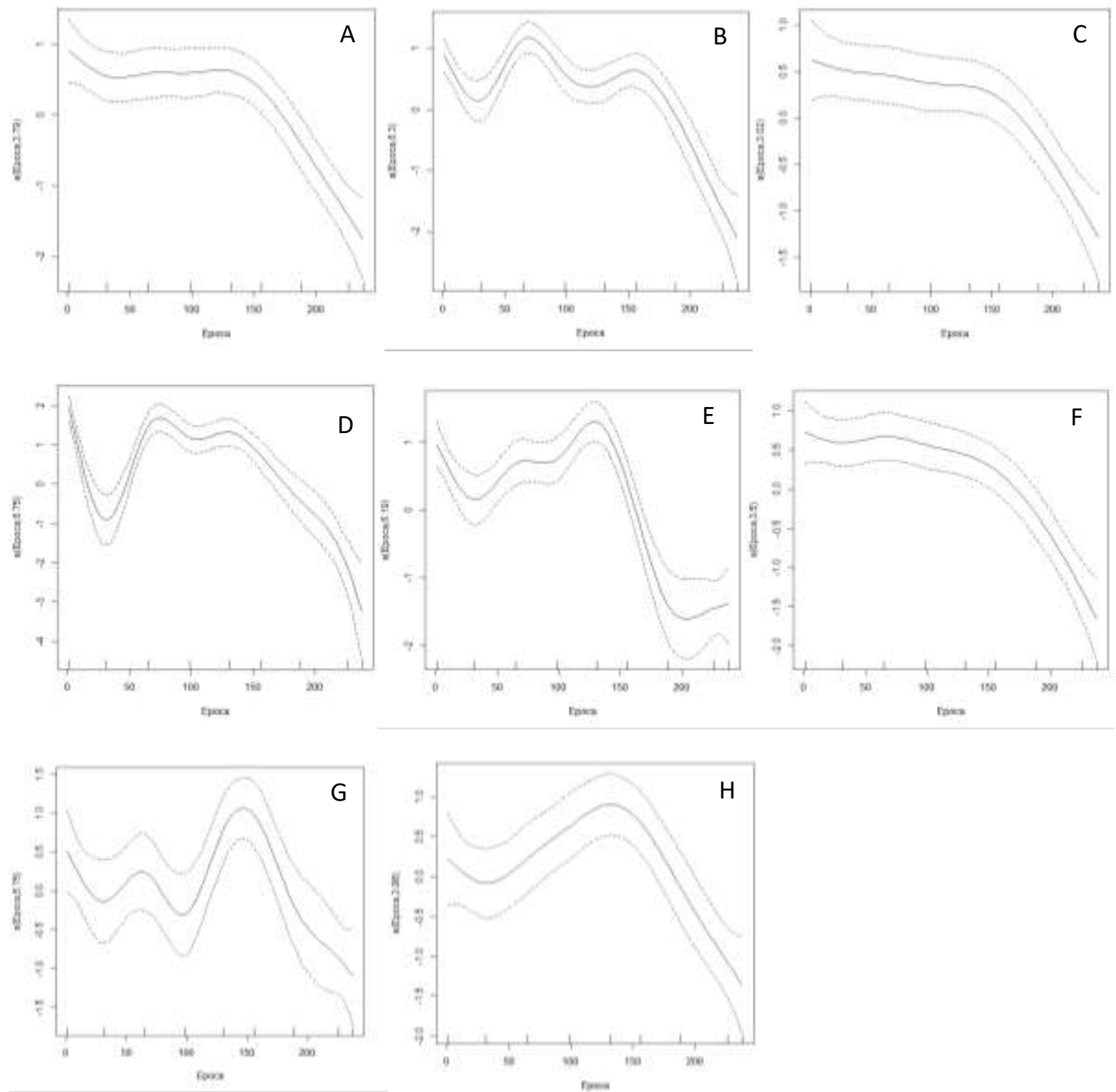

Fonte: Autores.

Durante o período de incidência do fungo C. coffeicola, a cultivar Catuaí Vermelho IAC 99 apresentou oscilações na formação de lesões. Aos 25 dias observou-se ponto mínimo de incidência, com aumento crescente chegando ao máximo de incidência aos 65 dias, correspondente ao mês de fevereiro (Figura 2). Este comportamento pode ser justificado pela relação de umidade relativa e temperatura observada durante os meses (Figura 1). Em fevereiro foi possível notar que a umidade relativa se manteve alta com temperaturas elevadas favorecendo a incidência. Entre os 100 e 130 dias de início das avaliações foi observado outro ponto mínimo de incidência, relacionado com a baixa umidade encontrada para o mês de maio, seguido de um leve aumento e posterior decréscimo até o final das avaliações.

As cultivares Bourbon Amarelo IAC J10, Topázio MG-1190 e IAC 125 RN, apresentaram queda inicial na incidência, próximo aos 20 dias de avaliação, fato não observado nos demais materiais genéticos.

Diferentes níveis de resistência à cercosporiose são verificados em progênies oriundas de cruzamentos com Caturra 
Vermelho ou Amarelo, dentre elas a cultivar Catuaí Vermelho IAC 144 (Fernandes et al., 1990). No entanto, essas progênies exibem grande variabilidade no campo.

Para a cultivar Acauã Novo a incidência de cercosporiose foi constante no período avaliado. Os valores mais altos foram encontrados durante os primeiros 110 dias de avaliação, em sequência esses valores diminuíram gradativamente (Figura 2). O mesmo comportamento foi observado para Acaiá Cerrado MG 1474 (Tabela 2).

A cultivar Bourbon Amarelo apresentou maior variação durante todo o período de avaliação, permitindo afirmar que a incidência de cercosporiose nesta cultivar é fortemente influenciada pelas condições climáticas. Aos 35 dias de avaliação foi observada a menor incidência de cercosporiose, coincidindo com um período de umidade relativa de $60 \%$ (Figura 1). No final das avaliações a ocorrência de cercosporiose foi quase nula. Carvalho et al. (2017) tem indicado cultivares do grupo Bourbon somente para cafeicultores que desejam obter um produto diferenciado em relação à qualidade de bebida, visto que essa cultivar apresenta menor produtividade, sendo mais exigente em nutrição, e mais suscetível às principais doenças do cafeeiro, inclusive a cercosporiose.

A maior incidência de cercosporiose na cultivar Topázio MG-1190 foi observada aos 150 dias, correspondente ao mês de maio. A cultivar IAC $125 \mathrm{RN}$ apresentou menor número de folhas lesionadas principalmente no início das avaliações, comportamento esse distinto das demais cultivares. O pico máximo de incidência foi observado entre os 120 e 140 dias (Figura 2), nos quais as condições climáticas apresentaram-se favoráveis ao desenvolvimento do fungo.

\subsection{Incidência de bicho-mineiro-do-cafeeiro}

Pela análise de Deviance (Tabela 3), verifica-se efeito significativo de todos os fatores analisados para número de folhas minadas. A interação dos fatores cultivares e épocas de avaliação foi significativa $(p<0,001)$, o que evidencia a dependência entre os fatores analisados neste experimento.

Tabela 3. Análise de Deviance para número de folhas minadas em cultivares de cafeeiros avaliados a cada 15 dias durante os meses de dezembro/2017 a agosto/2018 em Monte Carmelo, Minas Gerais.

\begin{tabular}{cccc}
\hline FV & GL & Deviance & valor-p \\
\hline Cultivar & 7 & $4,2 \times 10^{6}$ & $<0,001^{* *}$ \\
Época & 16 & $6,9 \times 10^{5}$ & $<0,001^{* *}$ \\
Bloco & 3 & $5,2 \times 10$ & $<0,01^{*}$ \\
Cultivar x Época & 112 & $4,2 \times 10^{6}$ & $<0,001^{* *}$
\end{tabular}

* significativo ao nível de $1 \%$ de probabilidade, ** significativo ao nível de $0,1 \%$ de probabilidade. FV: Fonte de Variação; GL: grau de liberdade. Fonte: Autores.

Nos meses de abril e maio de 2018, a cultivar Paraíso MG H 419-1 apresentou menor incidência média de folhas minadas em relação aos materiais genéticos Mundo Novo IAC 379-19, Catuaí Vermelho IAC 99, Bourbon Amarelo IAC J10, Topázio MG-1190 e IAC 125 RN (Tabela 4). Em junho de 2018, a cultivar Mundo Novo IAC 379-19 apresentou menor incidência da praga em relação as demais, não diferindo significativamente da cultivar Acaiá Cerrado MG-1474 (Tabela 4). Em julho, período em que as condições climáticas favorecem o inseto, a cultivar que apresentou menor quantidade de folhas minadas foi o Mundo Novo IAC 379-19. No mês seguinte, em 28/08/2018, foi detectada menor incidência da praga na cultivar Bourbon Amarelo IAC J10 quando comparado aos demais materiais genéticos testados, exceto Acauã Novo e Acaiá Cerrado MG-1474, as quais não diferiram significativamente entre si. 
As cultivares Paraíso MG H 419-1 e Topázio MG-1190 apresentam similaridade genética por possuírem como parental em comum uma cultivar do grupo Catuaí. Já o Acaiá Cerrado MG-1474, por ser proveniente de uma seleção dentro do grupo Mundo Novo, possui características semelhantes à cultivar de origem. Um dos parentais que originou a cultivar Mundo Novo é o Bourbon Vermelho. Diante disso, nota-se que nessas cultivares a incidência de bicho-mineiro foi menor quando comparada aos demais materiais genéticos. Entretanto, este fato isolado não confere resistência, mas explica a relação de menor incidência da praga entre essas cultivares.

Estudos conduzidos por Pereira (2011), com análise de resistência de clones ao bicho- mineiro e caracterização de genótipos de cultivares de C. arabica, por meio da ampliação de fragmentos genéticos e da genealogia formada entre os clones e as cultivares, observaram alta similaridade genética entre as cultivares Catuaí Vermelho IAC 144, Catuaí Amarelo IAC 62, Catucaí Vermelho 2015 cv.476, Catucaí Amarelo 2015 cv.479, Mundo Novo IAC 376-4, Obatã e Paraíso. Porém, apesar da similaridade genética encontrada, é importante destacar que estes materiais genéticos podem apresentar comportamentos agronômicos distintos.

Em experimento conduzido na região do Alto Paranaíba com distintos materiais genéticos, o número médio de minas intactas de bicho-mineiro-do-cafeeiro foi menor na cultivar Bourbon Amarelo em relação ao Acaiá e Paraíso (Rodrigues et al., 2014).

Convém ressaltar que em períodos anteriores às avaliações, foram realizadas aplicações de inseticidas químicos na área experimental. Assim, os resultados observados possivelmente podem ser decorrentes de diferenças existentes entre as cultivares.

Considerando este fato, a cultivar que apresentou maior incidência média de minas em todas as avaliações foi a IAC 125 RN, apresentando como principal característica resistência à ferrugem do cafeeiro (Tabela 4). Conceição et al. (2005), verificaram em experimento conduzido com cultivares de cafeeiros com diferentes níveis de resistência à ferrugem que maiores porcentagens de folhas com lesões de bicho-mineiro (63\% - 67\%) foram observadas entre julho e outubro de $2003 \mathrm{em}$ materiais resistentes à ferrugem - Tupi IAC 1669-33 e Obatã IAC 1669-20. Os autores justificaram que ocorreu uma retenção foliar dessas cultivares em função da resistência à doença e por consequência a porcentagem de folhas lesionadas pelo bichomineiro foi maior. Neste contexto, embora as cultivares Tupi e Obatã apresentassem alta porcentagem de folhas lesionadas, podem ter maior tolerância ao bicho-mineiro devido à menor queda de folhas. Diante disto, para explicar esta relação com a cultivar IAC $125 \mathrm{RN}$ seriam necessários estudos voltados para área foliar correlacionada com a resistência da ferrugem e a incidência de bicho-mineiro, uma vez que tal informação não se encontra disponível na literatura e não foi objetivo do presente estudo. 
Tabela 4. Incidência Média (IM) e Erro Padrão (EP) de folhas minadas no período de 21/12/2018 à 28/08/2018 em oito cultivares de cafeeiros.

\begin{tabular}{|c|c|c|c|c|c|c|c|c|c|}
\hline Cultivar & $\begin{array}{c}21 / 12 / 2017 \\
\mathrm{IM} \pm \mathrm{EP}\end{array}$ & $\begin{array}{c}08 / 01 / 2018 \\
\mathrm{IM} \pm \mathrm{EP}\end{array}$ & $\begin{array}{c}21 / 01 / 2018 \\
\mathrm{IM} \pm \mathrm{EP}\end{array}$ & $\begin{array}{c}10 / 02 / 2018 \\
\mathrm{IM} \pm \mathrm{EP}\end{array}$ & $\begin{array}{c}22 / 02 / 2018 \\
\mathrm{IM} \pm \mathrm{EP}\end{array}$ & $\begin{array}{c}09 / 03 / 2018 \\
\mathrm{IM} \pm \mathrm{EP}\end{array}$ & $\begin{array}{c}27 / 03 / 2018 \\
\mathrm{IM} \pm \mathrm{EP}\end{array}$ & $\begin{array}{c}10 / 04 / 2018 \\
\mathrm{IM} \pm \mathrm{EP}\end{array}$ & $\begin{array}{c}27 / 04 / 2018 \\
\mathrm{IM} \pm \mathrm{EP}\end{array}$ \\
\hline $\begin{array}{l}\text { Mundo } \\
\text { Novo }\end{array}$ & $5,7 \mathrm{a} \pm 4,0$ & $3,7 \mathrm{a} \pm 2,2$ & $0,7 \mathrm{a} \pm 1,2$ & $3,7 \mathrm{a} \pm 1,9$ & $0,5 \mathrm{a} \pm 5,5$ & $3,7 \mathrm{a} \pm 2,2$ & $5,2 \mathrm{a} \pm 3,5$ & $2,2 b \pm 5,6$ & $\begin{array}{c}12,0 \mathrm{a} \pm \\
6,7\end{array}$ \\
\hline $\begin{array}{c}\text { Catuaí } \\
\text { Vermelho }\end{array}$ & $2,5 \mathrm{a} \pm 2,0$ & $5,0 \mathrm{a} \pm 3,0$ & $0,5 \mathrm{a} \pm 8,6$ & $2,2 \mathrm{a} \pm 1,2$ & $0,2 \mathrm{a} \pm 3,0$ & $5,0 \mathrm{a} \pm 3,0$ & $2,0 \mathrm{a} \pm 1,3$ & $3,2 \mathrm{c} \pm 8,0$ & $\begin{array}{c}12,0 \mathrm{a} \pm \\
7,0\end{array}$ \\
\hline $\begin{array}{l}\text { Acauã } \\
\text { Novo }\end{array}$ & $\begin{array}{c}15,0 \mathrm{a} \pm \\
7,3\end{array}$ & $4,8 \mathrm{a} \pm 1,2$ & $0,7 a \pm 9,9$ & $1,0 \mathrm{a} \pm 1,4$ & $1,0 \mathrm{a} \pm 8,4$ & $5,0 \mathrm{a} \pm 1,2$ & $5,2 \mathrm{a} \pm 3,4$ & $\begin{array}{c}3,7 \text { abcd } \pm \\
2,6\end{array}$ & $\begin{array}{c}14,0 \mathrm{a} \pm \\
8,2\end{array}$ \\
\hline $\begin{array}{l}\text { Bourbon } \\
\text { Amarelo }\end{array}$ & $5,0 \mathrm{a} \pm 3,3$ & $5,1 \mathrm{a} \pm 3,0$ & $0,7 \mathrm{a} \pm 1,2$ & $1,2 \mathrm{a} \pm 7,6$ & $0,2 \mathrm{a} \pm 3,2$ & $5,1 \mathrm{a} \pm 3,0$ & $6,1 \mathrm{a} \pm 4,0$ & $4,2 \mathrm{~d} \pm 1,0$ & $7,4 \mathrm{a} \pm 4,2$ \\
\hline Paraíso & $\begin{array}{c}17,0 \mathrm{a} \pm \\
1,2\end{array}$ & $6,6 \mathrm{a} \pm 4,0$ & $2,7 \mathrm{a} \pm 4,1$ & $3,7 \mathrm{a} \pm 2,0$ & $0,5 \mathrm{a} \pm 5,1$ & $6,6 \mathrm{a} \pm 4,0$ & $2,0 \mathrm{a} \pm 1,4$ & $1,7 \mathrm{a} \pm 4,2$ & $\begin{array}{c}11,0 \mathrm{a} \pm \\
6,10\end{array}$ \\
\hline $\begin{array}{c}\text { Acaiá } \\
\text { Cerrado }\end{array}$ & $2,2 \mathrm{a} \pm 9,4$ & $3,3 \mathrm{a} \pm 1,3$ & $0,7 \mathrm{a} \pm 4,0$ & $1,3 \mathrm{a} \pm 2,0$ & $1,2 \mathrm{a} \pm 6,4$ & $3,3 \mathrm{a} \pm 1,3$ & $2 \mathrm{a} \pm 3,6$ & $\begin{array}{c}3,0 \text { abcd } \pm \\
1,2\end{array}$ & $6,3 \mathrm{a} \pm 2,1$ \\
\hline Topázio & $\begin{array}{c}11,0 \mathrm{a} \pm \\
7,3\end{array}$ & $6,0 \mathrm{a} \pm 3,4$ & $0,0 \mathrm{a} \pm 5,0$ & $3,7 \mathrm{a} \pm 2,0$ & $0,5 a \pm 5,1$ & $6,0 a \pm 3,4$ & $2,2 \mathrm{a} \pm 1,2$ & $2,7 c \pm 6,4$ & $8,4 \mathrm{a} \pm 4,7$ \\
\hline $\begin{array}{c}\text { IAC } 125 \\
\text { RN }\end{array}$ & $\begin{array}{c}36,0 \mathrm{a} \pm \\
2,4 \\
\end{array}$ & $1,3 \mathrm{a} \pm 7,2$ & $2,5 \mathrm{a} \pm 3,7$ & $5,0 \mathrm{a} \pm 2,5$ & $2,0 \mathrm{a} \pm 1,6$ & $1,3 \mathrm{a} \pm 7,2$ & $1,2 \mathrm{a} \pm 9,3$ & $4,3 \mathrm{~d} \pm 1,0$ & $\begin{array}{c}14,0 \mathrm{a} \pm \\
8,2 \\
\end{array}$ \\
\hline Cultivar & $\begin{array}{c}13 / 05 / 2018 \\
\mathrm{IM} \pm \mathrm{EP}\end{array}$ & $\begin{array}{c}21 / 05 / 2018 \\
\mathrm{IM} \pm \mathrm{EP}\end{array}$ & $\begin{array}{c}11 / 06 / 2018 \\
\mathrm{IM} \pm \mathrm{EP}\end{array}$ & $\begin{array}{c}21 / 06 / 2018 \\
\mathrm{IM} \pm \mathrm{EP}\end{array}$ & $\begin{array}{c}07 / 07 / 2018 \\
\mathrm{IM} \pm \mathrm{EP}\end{array}$ & $\begin{array}{c}28 / 07 / 2018 \\
\mathrm{IM} \pm \mathrm{EP}\end{array}$ & $\begin{array}{c}10 / 08 / 2018 \\
\mathrm{IM} \pm \mathrm{EP}\end{array}$ & $\begin{array}{c}28 / 08 / 2018 \\
\mathrm{IM} \pm \mathrm{EP}\end{array}$ & - \\
\hline $\begin{array}{l}\text { Mundo } \\
\text { Novo }\end{array}$ & $\begin{array}{c}24,0 \mathrm{~cd} \pm \\
6,1\end{array}$ & $0,7 \mathrm{a} \pm 1,2$ & $\begin{array}{c}10,7 \mathrm{a} \pm \\
2,7\end{array}$ & $a \pm 4,5$ & $\begin{array}{c}10,4 \mathrm{a} \pm \\
3,2\end{array}$ & $\begin{array}{c}24,4 \mathrm{a} \pm \\
1,2\end{array}$ & $3,7 b \pm 9,3$ & $\pm 2,1$ & - \\
\hline $\begin{array}{c}\text { Catuaí } \\
\text { Vermelho }\end{array}$ & $\begin{array}{c}22,0 \mathrm{c} \pm \\
5,5\end{array}$ & $0,5 a \pm 8,6$ & $\begin{array}{c}15,0 \mathrm{c} \pm \\
3,2\end{array}$ & $9,7 \mathrm{a} \pm 5,3$ & $\begin{array}{c}13,5 \mathrm{~b} \pm \\
4,0\end{array}$ & $\begin{array}{c}42,2 \mathrm{a} \pm \\
2,0\end{array}$ & $4,4 \mathrm{c} \pm 1,1$ & $5,0 \mathrm{a} \pm 3,0$ & - \\
\hline $\begin{array}{l}\text { Acauã } \\
\text { Novo }\end{array}$ & $\begin{array}{c}20,0 \text { abcde } \\
\quad \pm 9,3\end{array}$ & $\begin{array}{c}0,7 \mathrm{a} \pm \\
10,0\end{array}$ & $\begin{array}{l}18,0 \text { abcd } \\
\pm 8,5\end{array}$ & $\begin{array}{c}13,3 \mathrm{a} \pm \\
7,2\end{array}$ & $\begin{array}{c}14,3 \text { abcd } \\
\pm 4,2\end{array}$ & $\begin{array}{c}37,1 \mathrm{a} \pm \\
2,0\end{array}$ & $\begin{array}{c}5,2 \text { abcd } \pm \\
3,2\end{array}$ & $3,5 \mathrm{a} \pm 2,2$ & - \\
\hline $\begin{array}{l}\text { Bourbon } \\
\text { Amarelo }\end{array}$ & $\begin{array}{c}20,0 \mathrm{~b} \pm \\
5,0\end{array}$ & $0,7 \mathrm{a} \pm 1,2$ & $\begin{array}{c}13,0 \mathrm{~b} \pm \\
3,7\end{array}$ & $9,0 \mathrm{a} \pm 4,7$ & $\begin{array}{c}16,5 \mathrm{c} \pm \\
4,8\end{array}$ & $\begin{array}{c}42,0 \mathrm{a} \pm \\
2,0\end{array}$ & $1,2 \mathrm{a} \pm 3,2$ & $3,3 \mathrm{a} \pm 2,1$ & - \\
\hline Paraíso & $\begin{array}{c}14,0 \mathrm{a} \pm \\
3,5\end{array}$ & $2,7 \mathrm{a} \pm 4,1$ & $\begin{array}{c}17,0 \mathrm{~d} \pm \\
4,2\end{array}$ & $\begin{array}{c}10,0 \mathrm{a} \pm \\
5,3\end{array}$ & $\begin{array}{c}16,7 \mathrm{c} \pm \\
5,0\end{array}$ & $\begin{array}{c}26,0 \mathrm{a} \pm \\
1,2\end{array}$ & $5,2 \mathrm{~d} \pm 1,3$ & $3,0 \mathrm{a} \pm 2,0$ & - \\
\hline $\begin{array}{c}\text { Acaiá } \\
\text { Cerrado }\end{array}$ & $\begin{array}{l}23,0 \text { abcde } \\
\quad \pm 6,5\end{array}$ & $0,7 \mathrm{a} \pm 4$ & $\begin{array}{l}16,5 \mathrm{abcd} \\
\pm 5,0\end{array}$ & $=3,0$ & $\begin{array}{c}20,0 \text { bcd } \pm \\
3,5\end{array}$ & $\begin{array}{c}29,7 \mathrm{a} \pm \\
8.1\end{array}$ & $\begin{array}{c}4,4 \text { abcd } \pm \\
1,6\end{array}$ & $4,3 \mathrm{a} \pm 1,5$ & - \\
\hline Topázio & $\begin{array}{c}28,0 \mathrm{e} \pm \\
7,0\end{array}$ & $0,0 \mathrm{a} \pm 5,0$ & $\begin{array}{c}12,7 \mathrm{~b} \pm \\
3,1\end{array}$ & $8,4 \mathrm{a} \pm 4,5$ & $\begin{array}{c}21,0 \text { ed } \pm \\
6,0\end{array}$ & $\begin{array}{c}35,4 \mathrm{a} \pm \\
2,0\end{array}$ & $5,0 \mathrm{~d} \pm 1,2$ & $5,1 \mathrm{a} \pm 3,0$ & - \\
\hline $\begin{array}{c}\text { IAC } 125 \\
\text { RN }\end{array}$ & $\begin{array}{c}24,0 \mathrm{~d} \pm \\
5,7\end{array}$ & $2,5 \mathrm{a} \pm 4,0$ & $\begin{array}{c}15,7 \mathrm{~d} \pm \\
4,0\end{array}$ & $\begin{array}{c}11,0 \mathrm{a} \pm \\
6,1\end{array}$ & $\begin{array}{c}21,0 \mathrm{~d} \pm \\
\quad 6,0\end{array}$ & $\begin{array}{c}29,6 \mathrm{a} \pm \\
1,4\end{array}$ & $4,1 \mathrm{c} \pm 9,7$ & $5,0 \mathrm{a} \pm 3,0$ & - \\
\hline
\end{tabular}

Médias seguidas pela mesma letra na coluna não diferem entre si pelo teste Tukey ao nível de 0,01\% de probabilidade. Fonte: Autores.

A ocorrência da praga foi verificada em todo o período amostrado. $\mathrm{O}$ aumento na incidência do bicho-mineiro foi observado a partir do mês de abril até meados de agosto, correspondendo a um período aproximado de 111 dias, exceto na última avaliação do mês de maio em que a incidência da praga foi praticamente nula. Este comportamento está relacionado com fatores climáticos, tais como a temperatura e a precipitação, os quais exercem papel fundamental na dinâmica populacional da praga (Silva et al., 2013; Amaral et al., 2015; Nunes et al., 2018).

O mês de julho apresentou maior número de minas devido às condições climáticas serem favoráveis ao desenvolvimento do inseto (Figura 1). Em conformidade com Rezende et al. (2017), o maior número de lesões se correlaciona negativamente com a precipitação e umidade relativa. $\mathrm{O}$ aumento do número de minas ocorre depois de um período sem chuvas e o decréscimo é no período chuvoso. Este comportamento foi observado para a avaliação no mês de agosto, no qual houve aumento da precipitação e temperaturas mais amenas.

Outros fatores também exercem papel fundamental na dinâmica populacional da praga, como presença de predadores e plantas daninhas (Souza et al., 2014). Nesse contexto, Lomelí-Flores et al. (2010) avaliando os impactos do clima na 
dinâmica populacional de bicho-mineiro e seus inimigos naturais no México, verificaram que o aumento populacional da praga em certos períodos pode ter ocorrido, em parte, às variações na temperatura ambiente entre as avaliações.

O desdobramento do fator épocas dentro de cada cultivar foi realizado por meio de Modelos Aditivos Generalizados (MAG). O dia zero corresponde ao início das avaliações (21/12/2018) e a data de 28/08/2018 corresponde aos 251 dias finais de avaliação (Figura 3).

Figura 3. Comportamento da incidência de minas em cultivares de cafeeiros: (A) Mundo Novo IAC 379-19; (B) Catuaí Vermelho IAC 99; (C) Acauã Novo; (D) Bourbon Amarelo IAC J10; (E) Paraíso MG H 419-1; (F) Acaiá Cerrado MG 1474; (G) Topázio MG 1190 e (H) IAC 125 RN.
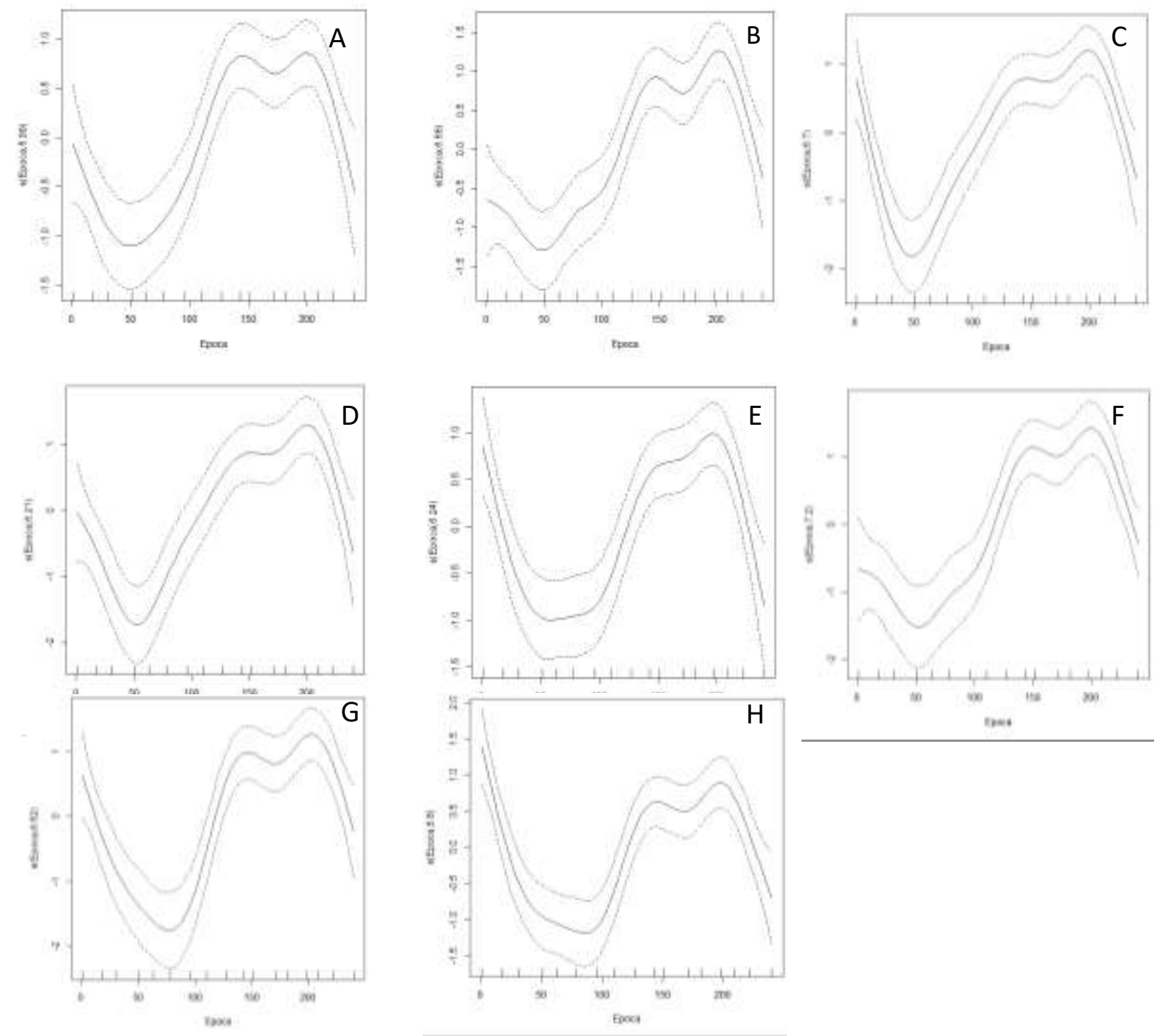

Fonte: Autores.

As cultivares apresentaram taxas mínimas de incidência da praga aos 50 dias de avaliação, ponto médio aos 130 dias e máximo entre 200 e 220 dias, o que pode ser justificado pelas condições meteorológicas durante o período de condução do experimento. A cultivar Mundo Novo IAC 379-19 apresentou baixa incidência da praga durante os 251 dias de avaliação, o que pode ser notado pelo valor negativo no eixo y do gráfico (Figura 3). Aos 130 dias, próximo à avaliação do dia 27/04/2018, observou-se um aumento das lesões e posteriormente uma leve queda, comportamento este justificado pelo período antecedente apresentar dias chuvosos (Figura 1), o que diminuiu as condições favoráveis ao desenvolvimento do inseto (Cintra, Filho \& Pereira, 2017). 
O número de folhas minadas para Catuaí Vermelho IAC 99 foi baixo no início das avaliações (Figura 3 e Tabela 4). Durante os primeiros 100 dias ocorreram oscilações na presença do inseto, influenciada justamente pela frequência de chuvas variadas durante este período. Portanto, pode-se afirmar que a incidência de lesões para esta cultivar nos meses de janeiro ao final de março de 2018 foi fortemente influenciada pelo índice pluviométrico. O maior número de lesões se deu aos 220 dias (correspondente à data de avaliação de 28/07/2018), apresentando média de 42,2 folhas minadas na parcela (Tabela 4).

Segundo Michelotto et al. (2006), Catuaí e Mundo Novo são as principais cultivares de C. arabica igualmente atacadas pelo inseto. No entanto, folhas lesionadas por L. coffeella são menos numerosas e visíveis nas plantas de Catuaí e Mundo Novo, pois tendem a se desprender dos ramos em função da incidência da ferrugem ( $H$. vastatrix) (Conceição et al., 2005).

A cultivar Paraíso MG H 419-1 apresentou incidência mínima da praga entre os 45 e 90 dias (Figura 3), correspondendo aos meses de fevereiro e março. O número de minas foi superior aos encontrados para Catuaí Vermelho, Acauã e Bourbon Amarelo no mesmo período.

O desempenho da cultivar Acaiá Cerrado MG-1474 ao longo do período de avaliação do experimento foi semelhante ao Catuaí Vermelho IAC 99, porém, com a diferença que a incidência da praga foi maior na cultivar Acaiá Cerrado. O mesmo comportamento foi observado por Moura et al. (2013) em experimentos com cultivares de cafeeiros no sistema de cultivo orgânico em três municípios de Minas Gerais, nos quais as cultivares Catuaí Vermelho IAC 99 e Paraíso MG H 419-1 apresentaram menor incidência da praga quando comparadas ao Acaiá Cerrado MG-1474.

Para a cultivar Topázio MG-1190 foi detectado maior número de minas no início da avaliação, posteriormente, houve diminuição da população da praga aos 75 dias. Como já relatado, neste período a temperatura média oscilou entre 20 e $25^{\circ} \mathrm{C}$ (Figura 1) e os índices de precipitação encontraram-se bastante altos, desfavorecendo o desenvolvimento do ciclo do bichomineiro.

A cultivar IAC $125 \mathrm{RN}$ apresentou maior incidência de bicho-mineiro principalmente nos primeiros 100 dias de avaliação. Após este período houve uma diminuição, chegando a apresentar valores nulos. O aumento ocorreu aos 220 dias do início das avaliações, sendo precedido por um período sem precipitação e de temperaturas elevadas (Figura 1).

Portanto, foi possível perceber que a incidência cresceu à medida que os fatores ambientais, tais como temperatura $\mathrm{e}$ precipitação, se tornaram favoráveis ao inseto, comportamento este observado em todas as cultivares. Assim sendo, destaca-se o mês de março com o menor número de lesões e os meses de junho e julho com os maiores. Em agosto, aos 230 dias, a presença de minas intactas foi reduzida em virtude do aumento das precipitações e da umidade em toda a área experimental.

\section{Conclusão}

As cultivares que apresentaram menor incidência de bicho-mineiro foram Mundo Novo IAC 379-19, Acaiá Cerrado MG-1474, Bourbon Amarelo IAC J10, Paraíso MG H 419-1 e Topázio MG-1190. A maior incidência de bicho-mineiro ocorreu no período de abril até meados de agosto.

A maior incidência de cercosporiose ocorreu no período de dezembro a maio, havendo variabilidade da ocorrência da doença entre as cultivares testadas.

\section{Referências}

Amaral, T. S., Bulhões, R. S., Paiva, T. S. S., \& Macêdo, J. A. (2015). Variação de dois componentes climáticos nas entrelinhas e em folhas de cafeeiro infestadas pelo bicho-mineiro. Magistra. 27(3/4), 344-352.

Carvalho, A. M. D., Mendes, A. N. G., Carvalho, G. R., Botelho, C. E., Gonçalves, F. M. A., \& Ferreira, A. D. (2010). Correlação entre crescimento e produtividade de cultivares de café em diferentes regiões de Minas Gerais, Brasil. Pesquisa Agropecuária Brasileira. 45(3), $269-275$. 
Carvalho, A. M. D. et al. (2017). Comportamento de cultivares de cafeeiro sob a incidência das doenças da ferrugem e cercosporiose em dois ambientes de cultivo. Coffee Science. 12(1), 100-107.

Cintra, P. H. M., Filho, A. B. E., \& Pereira, W. (2017). Flutuação populacional do bicho mineiro do cafeeiro em Ceres-GO. Congresso estadual de iniciação científica e tecnologia do IF goiano, 6.

Companhia Nacional de Abastecimento [CONAB] (2021, janeiro). Acompanhamento da safra brasileira de café - Terceiro levantamento. Recuperado de https://www.conab.gov. br/info-agro/safras/cafe

Companhia Nacional de Abastecimento [CONAB] (2020, setembro). Acompanhamento da safra brasileira de café - Terceiro levantamento. Recuperado de https://www.conab.gov. br/info-agro/safras/cafe

Conceição, C. H. C., Filho, O. G., \& Gonçalves, W. (2005). Flutuação populacional do bicho-mineiro em cultivares de café arábica resistentes à ferrugem. Bragantia. 64(4), 625-631.

Felipe, C. R. de P., Camarano, L. F., Oliveira, C. A. S., \& Ribeiro, D. A. (2005). Intensidade de doenças e pragas em plantas recepadas de café (Coffea arabica L.) cultivadas em três espaçamentos durante o segundo ano de produção. Simpósio de Pesquisa dos Cafés do Brasil, 4.

Fernandes, A. T. F., et al. (1990). Resistência de progênies de Catimor a diferentes isolados de Cercospora coffeicola Berk. \& Look. Fitopatologia Brasileira. $15,45-49$.

Fernandes, M. I. S., Assis, G. A., Nascimento, L. G., Cunha, B. A., Airão, A. L. C., \& Gallet, D. S. (2020). Parâmetros produtivos e de qualidade de cultivares de cafeeiros na região do Alto Paranaíba, Minas Gerais, Brasil. Research, Society and Development. 9(9).

Lomelí-Flores, J. R., Barrera, J. F., \& Bernal, J. (2010). Impacts of weather shade cover and elevation on coffee leaf miner Leucoptera coffeella (Lepidoptera: Lyonetiidae) population dynamics and natural enemies. Crop Protection, 29, 1039-1048.

Mesquita, C. M. et al. (2016). Manual do café: distúrbios fisiológicos, pragas e doenças do cafeeiro (p. 62). Belo Horizonte: EMATER, MG.

Michelotto, M.D., Galli, J. A., Martins, A. L. M., Spatti, L. R., \& Fazuoli, L. C. (2006). Ocorrência do bicho- mineiro do cafeeiro em diferentes linhagens de Icatu. Reunião Anual do Instituto Biológico, 68, 470-473.

Moura, W. D. M., Lima, P. C., Fazuoli, L. C., Condé, A. B. T., \& Silva, T. C. (2013). Desempenho de cultivares de café em sistema de cultivo orgânico na zona da mata mineira. Coffee Science. 8(3), 256-264.

Nunes, J. F., Martins, F. K., Franklin, A. M., \& Costa, E. S. (2018). Inimigos naturais da Broca-do-Café e do Bicho Mineiro do Cafeeiro (Coffea arabica L.) em Passos, MG. Ciência ET Praxis. 11(21), 115-120.

Oliveira, F. R. A., \& Ghini, R. (2012). Incidência e severidade da ferrugem (Hemileia vastatrix) do cafeeiro em função do aumento da concentração de $\mathrm{CO}_{2}$ do ar. Workshop sobre mudanças climáticas e problemas fitossanitários. Embrapa Meio ambiente. 120-126.

Ortega, A. C., \& Jesus, C. M. (2011). Território café do Cerrado: transformações na estrutura produtiva e seus impactos sobre o pessoal ocupado. Revista de Economia e Sociologia Rural. 49(3), 771-800

Patricio, F. R. A., Braguini, M. T., \& Fazuoli, L. C. (2010). Resistência de plantas de Coffea arabica, Coffea canephora e híbridos interespecíficos à cercosporiose. Bragantia. 69(4), 883-890.

Pereira, A. S., Shitsuka, D. M., Parreira, F. J. \& Shitsuka, R. (2018). Metodologia da pesquisa científica. UFSM. https://repositorio.ufsm.br/bitstream/handle/1/15824/Lic_Computacao_Metodologia-Pesquisa-Cientifica.pdf?sequence=1.

Pereira, G. S. (2011). Análise da resistência ao bicho-mineiro e caracterização de genótipos de Coffea arabica com microssatélites. Dissertação - Curso de Agronomia. Universidade Federal de Lavras, Lavras, MG, Brasil.

Pozza, E. A., \& Alves, M. C. (2008). Impacto potencial das mudanças climáticas sobre as doenças fúngicas do cafeeiro no Brasil. In: Ghini, R., \& Hamada, E. (Ed.). Mudanças climáticas: impactos sobre doenças de plantas no Brasil (p. 216-233). Brasília: EMBRAPA.

Reis, P. R., \& Souza, J. C. (1996). Manejo integrado do bicho-mineiro Perileucoptera coffeella (Guérin-Mèneville, 1842) (Lepidoptera: Lyonetiidae), e seu reflexo na produção de café. Sociedade Entomológica do Brasil, 25, 77-82.

Rezende, A. N., Carvalho, S. J. P., \& Peixoto, M. L. (2017). Flutuação populacional do bicho-mineiro influenciada pelo controle de plantas daninhas nas entrelinhas do cafeeiro. Congresso brasileiro de pesquisas cafeeiras, 43.

Ribeiro, A. C., Guimarães, P. T. G., \& Alvarez, V. H. (1999). Recomendações para o uso de corretivos e fertilizantes em Minas Gerais. $5^{a}$ Aproximação, 18, 289-302). Viçosa, MG.

Rodrigues, B., Carvalho, F.J., Oliveira, D. F. M., Andaló, V., \& Assis, G.A. (2014). Ocorrência de bicho-mineiro em diferentes cultivares de cafeeiro na região do Alto Paranaíba. Congresso brasileiro de pesquisas cafeeiras, 40.

Rodrigues, W. N. et al. (2015). Severidade da cercosporiose em genótipos de café arábica em cultivo adensado. Congresso brasileiro de pesquisas cafeeiras, 41 .

Salgado, B. G., Macedo, R. L. G., Carvalho, V. L., Salgado, M., \& Venturin, N. (2007). Progresso da ferrugem e da cercosporiose do cafeeiro consorciado com grevílea, com ingazeiro e a pleno sol em Lavras, MG. Ciência e Agrotecnologia. 31(3), 1067-1074.

Scalon, J. D., Mateus, A. L. S. S., \& Zacarias, M. S. (2013). Análise espaço-temporal do nível de infestação do bicho-mineiro Leucoptera coffeella (GuérinMenèville \& Perrottet. 1842) (Lepidoptera: Lyonetiidae) em cafezal orgânico (Coffea arabica L.). Coffee Science. 8(3), 347-353. 
Research, Society and Development, v. 10, n. 3, e29210313304, 2021

(CC BY 4.0) | ISSN 2525-3409 | DOI: http://dx.doi.org/10.33448/rsd-v10i3.13304

Silva, M. D. C. et al. (2006). Coffee resistance to the main diseases: leaf rust and coffee berry disease. Brazilian Journal of Plant Physiology, $18,119-147$.

Silva, M. G. D. (2014). Interação da luz, da temperatura e do molhamento foliar em Cercospora coffeicola e na Cercosporiose em cafeeiro. Tese (Doutorado em Fitopatologia) - Universidade Federal de Lavras, Lavras, MG, Brasil.

Silva, R. A., Machado, J. L., Carvalho, T. A. F., Matos, C. S. M., \& Pereira, A.B. (2013, novembro). Influência das condições climáticas na flutuação populacional do bicho-mineiro do cafeeiro Leucoptera coffeella (Guérin-Mèneville, 1842) (Lepidoptera: Lyonetiidae) no sul de Minas Gerais. Simpósio de pesuisa dos caés do Brasil. 8 .

Sistema meteorológico COOXUPÉ [SISMET] (2018, julho). Recuperado de http://sismet.cooxupe.com.br:9000

Souza, V. C. O., Cunha, R. L., Andrade, L. N., Volpato, M. M. L., Carvalho, V. L., \& Esmin, A. A. A. (2013). Técnicas de extração de conhecimentos aplicadas à modelagem de ocorrência da cercosporiose (Cercospora coffeicola Berkeley \& Cooke) em cafeeiros na região sul de Minas Gerais. Coffee Science. $8(1), 91-100$.

Souza, T. P. et al. (2014). Ocorrência sazonal, predação e parasitismo de Leucoptera coffeella (Guérin-Méneville, 1842) (Lepidoptera: Lyonetiidae) em cafeeiros associados à grevíleas. Coffee Science. 9(1), 34-50.

Souza. A. G. C. et al. (2015). A time series analysis of brown eye spot progress in conventional and organic coffee production systems. Plant Pathology. 64(1), 157-166.

Zambolim, L., Vale, F. X. R., \& Zambolim, E. M. (2005). Doenças do cafeeiro. In: Kimati, H. et al. (Ed.). Manual de Fitopatologia: Doenças das plantas cultivadas. 2, 4, 165-180. 